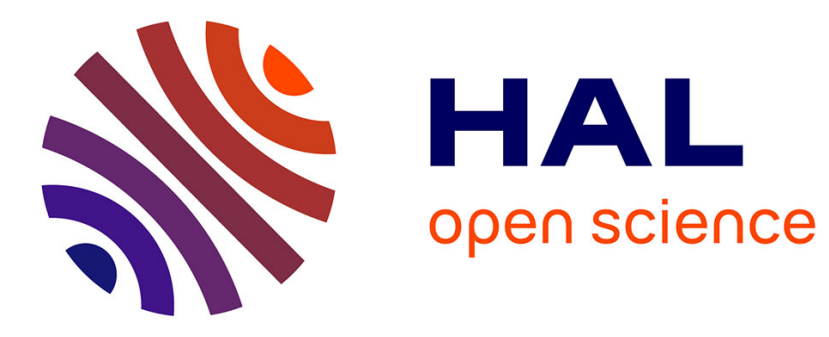

\title{
How quantum dots aggregation enhances Förster Resonant Energy Transfer
}

Julie Hottechamps, Thomas Noblet, Alain Brans, Christophe Humbert, Laurent Dreesen

\section{- To cite this version:}

Julie Hottechamps, Thomas Noblet, Alain Brans, Christophe Humbert, Laurent Dreesen. How quantum dots aggregation enhances Förster Resonant Energy Transfer. ChemPhysChem, 2020, 21 (9), pp.853 - 862. 10.1002/cphc.202000067 . hal-02497042

\section{HAL Id: hal-02497042 \\ https://hal.science/hal-02497042}

Submitted on 18 Nov 2020

HAL is a multi-disciplinary open access archive for the deposit and dissemination of scientific research documents, whether they are published or not. The documents may come from teaching and research institutions in France or abroad, or from public or private research centers.
L'archive ouverte pluridisciplinaire HAL, est destinée au dépôt et à la diffusion de documents scientifiques de niveau recherche, publiés ou non, émanant des établissements d'enseignement et de recherche français ou étrangers, des laboratoires publics ou privés. 


\title{
How quantum dots aggregation enhances Förster Resonant Energy Transfer
}

\author{
Julie Hottechamps ${ }^{* \dagger} \quad$ Thomas Noblet $^{\dagger} \quad$ Fabrice Bouillenne ${ }^{\ddagger}$ \\ Christophe Humbert ${ }^{\S} \quad$ Laurent Dreesen*†
}

\section{Abstract}

As luminescent quantum dots (QDs) are known to aggregate themselves through their chemical activation by carbodiimide chemistry and their functionalization with biotin molecules, we investigate the effects of both on the fluorescence properties of CdTe QDs and their influence on Förster Resonant Energy Transfer (FRET) occurring with fluorescent avidin molecules (FA). First, the QDs fluorescence spectrum undergoes significant changes during the activation step which are explained thanks to an original model. We also highlight the strong influence of biotin in solution on FRET efficiency, and define the experimental conditions maximizing the FRET. Finally, an aggregated-QD-based system and a free-QD-based system are studied in order to compare their limits of detection. The results show a detection threshold of $5 \mathrm{nM}$ in FA for the aggregated-QDbased system while it is equal to $80 \mathrm{nM}$ for the non-aggregated one, surprisingly attesting the superiority of the former.

\section{Introduction}

Research on the development of systems allowing the detection of biological interactions or the presence of specific analytes in biological environment has become a brand new trend for these last years. This stems from the constant evolution of technologies and the need for more and more powerful and accurate devices which have to respond instantaneously. A large part of biomedical research is then dedicated to the conception of new biosensors which present high specificity and affinity with biomolecules such as proteins, peptides or DNA.

\footnotetext{
*corresponding author

${ }^{\dagger}$ GRASP-Biophotonics, CESAM, University of Liege, Institute of Physics, Allée du 6 août 17, 4000 Liège, Belgium. E-mail: J.Hottechamps@uliege.be, Laurent.Dreesen@uliege.be

${ }^{\ddagger}$ Laboratory of Enzymology and Protein Folding, Center for Protein Engineering (CIP), University of Liege, Quartier Agora, Allée du six Août 11, B6a, 4000 Liège, Belgium

$\S$ Univ Paris-Sud, Université Paris-Saclay, Laboratoire de Chimie Physique, CNRS, Bâtiment 201P2, 91405 Orsay, France.
} 
The signal transduction method that can account for biological interactions is often based on optics because of its ease and rapidity of use, immunity to electromagnetic interference and the possibility to perform multiplexed detection $[1,2]$. Förster Resonance Energy Transfer (FRET) is one of the most popular phenomenon operating in optical biosensors nowadays. This non-radiative transfer of energy from an excited donor fluorophore to an acceptor fluorophore closely depends on the proximity of both entities, which makes FRET ideal for probing biological interactions [3, 4].

Thanks to the expansion of nanoscience over the years, semiconductor nanocrystals, also called quantum dots (QDs), gathered momentum and proved unavoidable in FRET based biosensors [5, 6]. These nanoparticles exhibit unique electronic $[7,8,9,10,11]$ and optical $[12,13,14,15]$ properties arising from their nanoscale dimensions which entail quantum confinement effects. QDs band gap energy is therefore size-dependent, driving to tunable emission spectra [16]. Their broad absorption spectra, robustness and high quantum yield as well as their long fluorescence lifetime combined to their narrow and tunable emission spectra, make QDs the best candidates over organic dyes as donors in FRET-based systems $[3,17,18,4,19]$. Plenty of these biosensors have already been studied for applications in various field [20] such as medical diagnostics $[4,19,21]$, bioanalytics $[22,23,24]$ or food industry [25]. The high surfaceto-volume ratio of QDs allows immobilization of several biomolecules on their surface leading to a rise of FRET efficiency and therefore a more sensitive detection system [4].

The main challenge throughout the design of a biosensor using QDs lies in the functionalization step which actually consists in tagging QDs with recognition molecules. Various procedures already exist in bioconjugation of QDs, each being specific to the target molecule $[26,20]$. Since QDs have to be water soluble for biological applications with biomolecules attached to their surface, they are coated with functional ligands in a previous step. The choice of ligands depends on the biomolecule and the transduction method involved in the sensing system. QDs are often coated with hydrophilic ligands but their poor stability is well known and usually needs to be avoided [27, 28]. Hence, many coating strategies consisting in the encapsulation of QDs in amphiphilic polymers have been developed and brought a significantly higher stability [29, 30,31]. Yet, QDs with hydrophilic ligands coating appear to be more appropriate because they exhibit a shorter hydrodynamic radius. Indeed, applications based on FRET require the minimization of this radius as it works only if QDs are close to the acceptor molecule [27, 32, 33].

Amongst the different techniques used for the bioconjugation of molecules onto QDs and metallic nanoparticles, carbodiimide chemistry has been widely employed and optimized for many years in order to link two given species both presenting amine or carboxylic acid functional groups [34, 35]. First, 1-ethyl3-(3-dimethylaminopropyl) carbodiimide (EDC) reacts with the carboxylic acid groups located at the QD surface to form a reactive intermediate $o$-acylisourea able to link with the primary amines of the biomolecules. Second, an amide bond is formed thanks to the addition of N-hydroxysuccinimide (NHS) esters or 
their sulfonated derivatives $[26,36,37,38,39]$. Despite the popularity of this method, built on its ease of use, an unsuitable QDs aggregation might occur subsequently to this chemistry [40, 26, 41]. Mattoussi et al. [42] even went as far as changing their functionalization strategy to get around this phenomenon. Although the effects of surface coating on QDs properties have been already partially studied $[32,43]$ as well as the impact of their bioconjugation [44], to our knowledge, there are only a few traces in literature of a description of the effects of carbodiimide chemistry on QDs optical properties [45], but not as complete and quantitative as the work we present here.

In order to address this issue, this work aims at giving a description of how QDs functionalization can affect the FRET phenomenon but also at studying why carbodiimide chemistry alters QDs optical properties. Thanks to fluorescence and UV-visible spectroscopies, this chemical process first lead to QDs aggregation, as confirmed by dynamic light scattering (DLS) measurements reported here. This phenomenon entails modifications in emission and extinction spectra of QDs which are explained using an analytical model based on intraaggregate screening and FRET between QDs. In accordance with the pioneer experimental design [36], EDC involved in this carbodiimide chemistry is usually used in excess $[26,32,37,38]$. This paper emphasizes the relationship between the QDs aggregation phenomenon and the amount of EDC and sulfoNHS reagents, showing that the carbodiimide chemistry is still effective by lowering their concentrations while avoiding aggregation. The most popular couple of molecules involved in a model system based on QDs functionalization is the biotin-avidin archetype complex due to the high affinity between both components $[19,26,46,47]$. In this work, streptavidin tagged with fluorophore is used to recognise biotinylated QDs and to give rise to FRET. By comparing FRET efficiency and sensitivity of two systems respectively based on aggregated and free QDs, we evidence that the aggregation phenomenon in this type of biosensor does not constitute a drawback as we could dread. On the contrary, the detection threshold on molecular recognition by FRET is improved by a factor of 16 when aggregation occurs, provided the latter is finely monitored through an adapted chemical protocol as detailed in this contribution.

\section{Results and Discussion}

\subsection{QDs activation step}

Fluorescence spectroscopy is used to scrutinize the influence of activation step (i.e. addition of EDC and sulfoNHS) on colloidal CdTe QDs fluorescence emission as shown in Figure 1a. Based on the literature which recommends an excess of EDC for performing the carbodiimide chemistry [26, 32, 37, 38], the amount of EDC and sulfoNHS in this activation step is first chosen to work with a ratio of 1600 and 400 molecules for 1 CdTe QD. Since there are about 200 ligands per CdTe QD [48, 49], the EDC is in excess of about a factor 10 in this case. As soon as these activators are added in solution, the emission spectrum of colloidal 
CdTe QDs experiences a spectral redshift and a decrease in intensity. The fluorescence spectrum continues to deform more and more slowly throughout the activation step, until it stops after 30 minutes. At the end of the QDs activation $\left(\mathrm{t}=30^{\prime}\right)$, a large redshift of $14 \mathrm{~nm}$ and a strong decrease of $62 \%$ of fluorescence emission are observed. The origin of these significant modifications has to be identified to clear up its effect on FRET phenomenon.

\subsubsection{Drop of fluorescence intensity}

According to the literature, the fluorescence intensity of the CdTe QDs capped with MPA is strongly affected by the $\mathrm{pH}$ of the solvent [48]. In our case, we noticed for instance that the increasing of $\mathrm{pH}$ translates into intensity fluctuations and a decreasing signal-to-noise ratio, which would hinder an accurate computation of FRET efficiency. For the experiments presented here, the $\mathrm{pH}$ monitoring of the solution throughout the activation step showed us that the $\mathrm{pH}$ remains stable between 7.3 and 7.7, which rejects the hypothesis of a change in the QDs fluorescence intensity due to a change in $\mathrm{pH}$.

As presented in Figure 1b, the evolution of QDs UV-visible spectrum over 30 minutes of activation evidences a levelling of QDs absorption band at 488 $\mathrm{nm}$ along with an increase of the extinction in the low wavelength region. The absorption of both EDC and sNHS cannot either explain this increase of the extinction around $300 \mathrm{~nm}$ or the change in shape of the UV-visible spectra over the range $300-600 \mathrm{~nm}$, as confirmed by the UV-visible spectra of these activators shown in the Supporting Information (Figure A.1). However, since the scattering cross section is directly proportional to $\lambda^{-4}$, with $\lambda$ the light wavelength [50], these observations reveal the formation of QD aggregates whose sizes are sufficient to significantly scatter light. The aggregation of colloidal QDs has already been observed [26, 41, 42]: it is related to surface charges and thus depends on the nature of ligands. In our case, the carboxylic-acidterminated ligands are initially deprotonated and negatively charged in water, as deduced from their zeta potential of $-36 \mathrm{mV}$, hence a very good stability of the colloidal solution before activation (Supporting Information, section B). Then, the charge of the ligands is modified throughout the activation step, which leads to aggregation, as recently described by Snee [40]. Besides, as depicted in Figure 1c, DLS measurements evidence over the time the emergence of larger and larger scattering centers. Indeed, aggregates with hydrodynamic radii up to $300 \mathrm{~nm}$ are detected at the end of the activation step whereas colloidal CdTe QDs size is initially evaluated at $3.4 \mathrm{~nm}$ (which corresponds to previous HRTEM analysis [51] wherein the size distribution of identical CdTe QDs was estimated at $3.4 \pm 0.6 \mathrm{~nm}$ ). In addition, the slight decrease of the absorbance at $488 \mathrm{~nm}$ (Figure 1b) is likely to translate a sedimentation of the QDs, here again compatible with the hypothesis of an aggregation.

Therefore, the drop of fluorescence intensity noticed in Figure 1a during QDs activation does stem from the aggregation process, which translates into a screening effect. Here we propose to account for this screening process thanks to the Beer-Lambert law which gives the excitation beam intensity $I(x)$ as a 
function of the penetration length $x$ :

$$
I(x)=I_{0} e^{-\sigma N x},
$$

where $I_{0}$ is the incident beam intensity, $\sigma$ the QDs extinction cross section and $N$ the QDs local density. The characteristic extinction length upon which the light turns off is then:

$$
\xi=(\sigma N)^{-1} .
$$

Using UV-visible measurements and the method described elsewhere [51], we deduce an extinction cross section of $\sigma=1.16 \times 10^{-19} \mathrm{~m}^{2}$ at an excitation wavelength of $310 \mathrm{~nm}$. Inside aggregates, QDs are supposed to be in contact so that:

$$
N=\frac{1}{d^{3}},
$$

with $d=3.4 \mathrm{~nm}$ the mean diameter of QDs. In this case, the extinction length of QD aggregates is $\xi=30 \mathrm{~nm}$. This implies that the QDs in the center of each aggregate, i.e. at a distance greater than $30 \mathrm{~nm}$ from its surface, only receive a small amount of light (or no light at all) and are so screened as schematically shown in Figure 2a.

The percentage of QDs screened in each aggregate is given by:

$$
\left(1-\frac{\xi}{D}\right)^{3} \text {. }
$$

Using the mean radius of aggregates $D=300 \mathrm{~nm}$ deduced from DLS measurements, a value of $73 \%$ is obtained. The global fluorescence intensity should drop to $73 \%$ whereas measurements give a loss of $62 \%$. This suggests that another phenomenon may occur concurrently and could be related to the bathochromic shift observed in QDs fluorescence spectrum as detailed below.

\subsubsection{Bathochromic shift of fluorescence spectrum}

The formation of agglomerates at high concentration of QDs could lead to inner filter effects which could eventually redshift the maximum fluorescence wavelength [51]. However, the evolution of UV-visible spectra in Figure 1b shows that there is no significant variation of the QDs extinction in the vicinity of $520 \mathrm{~nm}$. This means that the QDs fluorescence is unaffected by the inner filter effects and this hypothesis is swept away.

Actually, the spectral ranges of absorption and emission in QDs are closely related to the nanoparticle size through their radius, $R$. Taking into account the size dispersion of colloidal CdTe QDs, it is possible to find a couple of neighboring QDs of radii $R_{1}$ and $R_{2}$, respectively, so that the absorption wavelength, $\lambda_{a}\left(R_{1}\right)$, of the former coincides with the emission wavelength, $\lambda_{e}\left(R_{2}\right)$, of the latter. Given that QDs are close to each other and that their absorption and emission spectra significantly overlap, all the conditions to observe FRET phenomenon within agglomerates of QDs are gathered. This process has been highlighted by Kagan et al. in close-packed CdSe QD solids [52]. They have 
compared spectra of QD solids and those of QDs dispersed in solution. The redshift of the emission spectrum of QD solids was observed while absorption spectra of both samples were indistinguishable. They have shown that a size dispersion of only $1.5 \%$ could lead to a significant bathochromic shift. Since the magnitude of the redshift increases with the size dispersion in their research, the shift of $14 \mathrm{~nm}$ observed in the present work is likely to stem from the same phenomenon considering a size dispersion of approximatively $5.9 \%$. Indeed, in Figure $2 \mathrm{~b}$, the fit of the QDs fluorescence spectrum with a Gaussian curve gives a $\sigma$ parameter of $0.10 \mathrm{~nm}$ which is compared to the QDs mean radius of $1.69 \mathrm{~nm}$ (see Supporting Information, section C). Other publications have been focused on the FRET phenomenon between QDs of the same nature but of slightly different sizes in the form of solid systems [53, 54, 55, 56]. According to Mayilo et al. [57], the bathochromic shift of the emission peak of CdTe QDs is also observed in solution. They electrostatically bound nominally single-sized QDs with calcium ions to form clusters in which FRET is possible [57]. A review dedicated to FRET between QDs even mentions that this redshift of QDs emission spectrum is the hallmark of what they call a homotransfer, i.e. an energy transfer within a nominally monochromatic (i.e. single-sized) QDs population [58].

In our case, the description and the implementation of this process is carried out to give a quantitative account of such a FRET between aggregated CdTe QDs in solution. The details of this method are given in the Supporting Information (section D), while the results of this model, combined with the screening effect, is displayed in Figure 2b. As mentioned in the Supporting Information (section D), the only fitting parameter in this modelling is the $a(r)$ coefficient which corresponds to a FRET coupling constant encoding the spatial dependance of the transfer constant $k_{T} \propto a(r) \propto 1 / r^{6}$. It is all the higher that the agglomerates are dense and compact. In this case, the bathochromic shift of 14 $\mathrm{nm}$ is obtained for $a(r)=1.5$. As shown in Figure $2 \mathrm{~b}$, the predicted level of fluorescence intensity matches the measurements. However, the appearance of the fluorescence band at the largest wavelengths $(540-580 \mathrm{~nm})$ does not entirely fit the experimental spectrum. As mentioned by Kagan et al., the energy transfer from small to large QDs enhances this red tails of the fluorescence and they actually also failed to reproduce it with a gaussian distribution [52]. The fact that the size dispersions of both QDs and their aggregates are not taken into account could explain the divergence in this case. Even for the screening effect, $\xi$ and $D$ are considered as mean values, although $\xi$ is specific to each QD and $D$ to each aggregate. Therefore, instead of enforcing a global drop of $73 \%$ on the entire spectrum, applying a different attenuation factor for each emission wavelength could lead to a better fit.

\subsection{QDs biotinylation step}

The evolution of the fluorescence spectrum of QDs during the biotinylation step (i.e. addition of biotin) is presented in Figure 3. In this process, the biotin-toQD ratios $B_{3}$ and $B_{4}$ are compared (see Table 1). Following the biotin addition 
to the activated QDs, the fluorescence emission partially recovers its intensity and slightly redshifts in both cases. After 60 minutes, the fluorescence spectra stabilize themselves, which means the end of the biotinylation step is reached, and show an increase of the redshifts up to 3 and $4 \mathrm{~nm}$, combined with a recover of $43 \%$ and $62 \%$ of their intensities. Therefore, Figure 3 clearly attests to the change of emission properties of such biotinylated colloidal CdTe QDs and shows the importance to check the influence of QDs functionalization before carrying experiments.

A first attempt to highlight FRET phenomenon between functionalized colloidal CdTe QDs and FA was performed within the above-mentioned experimental conditions, i.e. $400: 1$ for the sulfoNHS/QD ratio and $B_{4}$ for the biotin/QD ratio, in the case of $1 \mathrm{FA}$ for $1 \mathrm{CdTe} \mathrm{QD}$. The fluorescence spectrum, shown in Figure 4a, reveals a decrease of colloidal CdTe QDs fluorescence emission of $16 \%$ which may suggest a FRET occurrence. Nevertheless, the fluorescence emission of unfunctionalized QDs in the presence of FA, displayed in Supporting Information (Figure E.1), also undergoes a slight decrease of $28 \%$ which contradicts the FRET hypothesis.

\subsubsection{Hypsochromic shift of fluoresence spectrum}

The fluorescence spectrum of unfunctionalized QDs with FA (see Supporting Information, Figure E.1) also exhibits a slight blueshift of $3 \mathrm{~nm}$ of the maximum emission wavelength. These observations suggest that QDs emission is absorbed by FA in solution, independently from any FRET phenomenon. This could explain the loss of the overall QDs fluorescence intensity and the decrease of the QDs fluorescence contribution above $520 \mathrm{~nm}$ leading to the hypsochromic shift. Moreover, the implementation of this absorption phenomenon (detailed in Supporting Information, section E) is suitable for describing it and then supports this hypothesis. Hereafter, the absorption of QDs emission by FA, arising as soon as QDs and FA are both mixed in solution, has to be considered in data interpretation.

\subsubsection{FRET detection}

The number of biotin molecules in excess in samples, prepared as mentioned in section 2.1, is huge compared to the number of available binding sites on CdTe QDs. In Figure 4b, we report a study of FRET efficiency as a function of the biotin/QD ratio. In this work, the loss of QDs fluorescence, computed as the relative difference between the fluorescence intensity of functionalized QDs, $I_{Q D}^{\circ}$, and that of functionalized QDs in the presence of FA, $I_{Q D}^{*}$, is used to monitor FRET phenomenon. The greater the loss of QDs fluorescence intensity, the higher the FRET efficiency:

$$
\eta=1-\frac{I_{Q D}^{*}}{I_{Q D}^{\circ}} .
$$

As shown in Figure 4c, by reducing the biotin-to-QD ratio by a factor of 10 ( $B_{3}$ instead of $B_{4}$ ), colloidal CdTe QDs fluorescence emission sharply decreases by 
$83 \%$ whereas the FA fluorescence band appears and displays a greater emission intensity than that of FA alone in solution (for an identical concentration of 1 $\mu \mathrm{M})$. The ratios between the fluorescence amplitudes of QDs and FA before and after the FRET process are similar (Figure 4c,d):

$$
\frac{I_{Q D}^{*}}{I_{Q D}^{\circ}} \approx \frac{I_{F A}^{\circ}}{I_{F A}^{*}}
$$

which indeed supports the hypothesis of such a FRET occurrence. The reduction of biotin/QD ratio leads to an increase of FRET efficiency until it reaches a maximum of $90 \%$ at the ratio $B_{1}$ (Figure $4 \mathrm{~b}$ ). Under this critical biotin/QD ratio, a loss of FRET efficiency is observed.

These results arise from the ability of streptavidin to fix free biotin molecules easier than that bound to the QDs because of steric hindrance, as suggested by additional gel electrophoresis measurements presented in Supporting Information (section F). Indeed, as soon as the biotin-to-QD ratio increases, a large amount of biotin molecules is fixed all around each QD and occupy the nearby space of biotin while the number of molecules in excess in solution increases with the ratio. For ease, the FA is therefore more inclined to link with biotin in solution so that FRET does not occur. By decreasing the biotin/QD ratio until reaching the minimum amount of biotin required to fully functionalize the QDs, we may limit the possibility of FA to link with the latter, and then increase the probability to give rise to FRET. In this case, given that the ratio $B_{1}$ corresponds to an optimum FRET, obtained with an sNHS/QD ratio of 4 , the number of fixed biotins likely ranges from 4 to 20 per QD. A separation process, such as size exclusion by gel filtration [31], to remove molecule excess could overcome this phenomenon and made FRET efficiency independent of biotin/QD ratio when biotin molecules are in excess. Such a technique is difficult to set up due to the small size of QDs and the importance to avoid any damage to ligands [59]. For this reason and with the idea to limit the excess of biotin molecules which inhibits FRET efficiency, the critical biotin/QD ratio $B_{1}$ was chosen for all the subsequent measurements.

\subsubsection{QDs activation without aggregation}

The measurements displayed up to now prove that the aggregation occurring throughout the activation step does not hinder the FRET between QDs and FA. A study of its role as a function of activators concentration is therefore carried out to determine if this effect is likely to increase or rather reduce FRET efficiency.

Thanks to fluorescence spectroscopy, the influence of activator/QD ratio on factors attesting to aggregation is reported in Figure 5a. As expected, the loss of fluorescence intensity and the bathochromic shift of the maximum fluorescence wavelength are less important when activators proportion decreases. Therefore, the smallest activator/QD ratio that we study is equivalent to 4 activators per $\mathrm{QD}$, and appears to limit as much as possible the QDs aggregation. 
We still have to check that the QDs activation in these conditions is sufficient to give rise to FRET between QD and FA: although we reduce the concentration of EDC/sulfoNHS to avoid aggregation, these activators are required for the biotinylation process. A measurement of FRET efficiency according to activator/QD ratio for two different biotin/QD ratios is presented in Figure 5b. With the higher biotin proportion $\left(B_{3}\right)$, the fluorescence intensity drop is weaker when activators concentration decreases, indicating a less effective FRET. On the other hand, FRET efficiency proves to be independent from activators proportion for the smaller biotin/QD ratio $\left(B_{1}\right)$. The QDs chemical surroundings for these different conditions is sketched in Figure 6. Knowing that each QD owns a number of ligands estimated at $200[48,49]$, for the biotin/QD ratio $B_{3}$, a large excess of biotin molecules is assumed to remain in solution regardless of activators concentration. The amount of biotin molecules fixed onto the QDs surface still increases with the proportion of activators and FRET efficiency is all the greater. However, FRET is limited by the propensity of FA to become filled more easily with free biotin molecules in this case. When QDs are functionalized with the second studied biotin/QD ratio $\left(B_{1}\right)$, there are merely 20 biotin molecules per QD in solution. At low concentrations in activators, an equivalent amount of biotin molecules is fixed onto QDs compared to the higher biotin/QD ratio. The binding of biotin is indeed driven by the concentration in activators. Nevertheless, the proportion of free biotin molecules in solution is far less reduced implying that FA has no more choice to bind with the biotin linked to QDs, which leads to a high FRET efficiency. As the amount of biotin molecules to fix is restricted, the increasing of the activators proportion drives to a similar situation. Hence, the activation step does not imply any significant effect for small biotin/QD ratio and biotin is the limiting factor in this case.

As shown in Figure 5b, the smallest activator/QD ratio of $4: 1$ combined to the biotin/QD ratio $B_{1}$ constitutes the optimum conditions for an efficient detection of FRET without QDs aggregation.

\subsubsection{FRET efficiency and detection limits}

Two systems compatible with FRET have been then investigated: the first one exhibits FRET between individually activated QDs and FA; the second one involves FRET between activated QDs and FA fixed at the periphery of QD aggregates. The FRET efficiency and sensitivity of these two systems are compared hereafter.

First, the evolution of QDs fluorescence spectrum throughout activation and functionalization steps has to be checked. A bathochromic shift and a drop of QDs fluorescence intensity is logically observed for the system based on QDs aggregation whereas no significant change is noted for the other system, proving the lack of aggregation. Second, the loss of QDs fluorescence intensity when FA is added in various concentrations to biotinylated QDs is reported in Figure 7 to evaluate FRET efficiency. In virtue of Equation (5), this drop of QDs fluorescence intensity conveys directly FRET efficiency, despite the slight loss of fluorescence intensity noticed with unfunctionnalized QDs which is only due 
to the absorption of QDs fluorescence by FA. As shown in Figure 7, FRET efficiency seems similar for both systems at high concentration in FA, even though the system without aggregation is slightly favoured at the highest FA concentration. Conversely, this advantage goes to the system based on QDs aggregation when FA concentration becomes weaker. It follows that the latter system offers the smallest limit of detection of $5 \mathrm{nM}$ in FA whereas it amounts to $80 \mathrm{nM}$ in FA for the other. Finally, since these systems are studied with the aim of designing biosensors, the sensitivity is an unmissable factor to assess. This study shows that the system with QDs aggregation is the most sensitive but acts in a smaller range of FA concentration (under $100 \mathrm{nM}$ ) than that based on FRET between QD and FA individually (under $1 \mu \mathrm{M}$ ).

The different behaviours of these two systems may also be understood in the light of Hill-Langmuir equation [60]. As derived in Supporting Information (section G), this model gives the following concentration-dependence for the FRET efficiency:

$$
\eta=\left(1+\left(\frac{r}{R_{0}}\right)^{6} \frac{\left(k_{d} /[\mathrm{FA}]\right)^{n}+1}{N}\right)^{-1},
$$

wherein $r=4.7 \mathrm{~nm}$ is the QD-FA donor-acceptor distance, $R_{0}=5.8 \mathrm{~nm}$ is the Förster radius, [FA] is the molar concentration of FA, $k_{d}$ is the microscopic dissociation constant associated to the $\mathrm{QD}(\mathrm{FA})_{n}$ complex, $N$ is the maximum number of fixed FAs per QD, and $n$ is the Hill coefficient related to the cooperative behaviour of FA within its complexation with QDs. Thus, Equation (7) is used to fit the experimental determination of the FRET efficiency, $\eta$, as drawn on Figure 7. For both set of measurements, we count three fitting parameters: $N, n$ and $k_{d}$. These are given in Table 2 , and especially show that the binding of FA onto the QDs is a cooperative process, since $n>1$. Besides, the sharper increasing of $\eta$ with [FA] in the case of aggregation translates into a significantly smaller dissociation constant $k_{d}$. It is as though the affinity of FA with biotinylated QDs was stronger in the case of QD aggregates.

This observation is actually consistent with what is happening throughout the biotinylation step. In the case of the aggregated-QD-based system, the biotin molecules are mainly fixed onto the surface of the outer QDs, not of those which are located inside the aggregates. In comparison, for the same initial QD/biotin ratio in solution (i.e. 20 biotins per QD), all the isolated QDs get coated with biotin molecules when they are not aggregated after activation. It means that the coverage of biotin on the outer surface of aggregates, in the first case, is greater than that on the surface of isolated QDs, in the second case. This is particularly true since aggregation occurs when there is a great number of activators (100 times more important than for dispersed QDs), thus fostering the binding of biotin. In other words, in the case of agglomerates, we fix more biotin molecules onto a smaller surface. Therefore, the apparent surface of QDs is denser in biotin in the case of the aggregated-QD-based system, hence its greater affinity with FA. 


\section{Conclusions}

Our work has reported QDs aggregation as a consequence of carbodiimide chemistry as it is commonly used to activate QDs surface ligands. This aggregation phenomenon has proved to lead to significant modifications in the QDs emission spectrum. The relevance of intra-aggregate screening effect and FRET between QDs have been demonstrated to explain both the decrease of fluorescence intensity and its spectral bathochromic shift. By decreasing the amount of chemical reagents, i.e. EDC and sulfoNHS, this aggregation effect can be avoided while maintaining QDs activation process sufficiently effective. The importance of paying attention to the functionalization method of QDs to prevent from wrong interpretations was also described.

FRET measurements have shown a limit of detection of $5 \mathrm{nM}$ in FA for the aggregated-QD-based system whereas the free-QD-based system leads up to 80 $\mathrm{nM}$. Furthermore, the first system is more sensitive than the latter in the low concentration range. Following these results, the QDs aggregation phenomenon becomes advantageous because it increases the detection threshold by a factor of 16 in concentration provided the QDs activation step is finely monitored.

\section{Experimental Section}

\subsection{Materials and CdTe QDs functionalization}

CdTe QDs were purchased from Sigma-Aldrich (Ref. 777935 CdTe core-type 25 $\mathrm{mg}$ ). They are provided through water-soluble powder and exhibit carboxylic acid functional groups $(\mathrm{COOH})$. The fluorescence band of these commercial CdTe QDs is located at $520 \mathrm{~nm}$ according to the manufacturer. Stock solutions of $2.0 \times 10^{-4} \mathrm{M}$ were prepared in Milli-Q water.

Colloidal CdTe QDs were activated by amine coupling reaction using EDC and sulfoNHS reagents (Amine Coupling Kit, GE Healthcare) with a 4:1 ratio in all experiments.

Activated colloidal CdTe QDs were biotinylated with a ratio of 20000 modified biotin molecules (EZ-Link Amine-PEG ${ }_{2}$-Biotin, Thermo Scientific) for 1 CdTe QD in first experiments. A scheme of the molecule is reported in the Supporting Information (Figure H.1). The biotin-to-QD ratio was further modified, as specified in Table 1, to study its influence on FRET efficiency. Nevertheless, for all experiments involving QDs functionalized with biotin molecules, the QDs have been activated beforehand. The entire functionalization process of the QDs is mapped in the Supporting Information (Figure H.1).

Fluorescent avidin was purchased from Sigma-Aldrich (Ref. 89045 ATTO550-Streptavidin) and is named as FA throughout the article. It consists of a streptavidin molecule marked with fluorophores called ATTO. As mentioned by the manufacturer, FA exhibits absorption and fluorescence bands at $554 \mathrm{~nm}$ and $576 \mathrm{~nm}$, respectively, allowing the spectral overlap between the emission spectrum of QDs and the absorption spectrum of FA, as required for FRET 
(see Supporting Information, Figure I.1). The computation of this overlap gives indeed a Förster radius of $58 \AA$, as detailed in the Supporting Information (section I). Furthermore, the apparent distance between the QD and the FA can be estimated based on the addition of the biotin size [61] with its spacer arm $\left(\mathrm{PEG}_{2}\right)$ (Thermo Scientific) and the ligands (MPA) [62] which gives approximately $47 \AA$ (see Supporting Information, section H). Such a distance is thus in keeping with the Förster radius of $58 \AA$.

\section{$5.2 \quad$ Fluorescence spectroscopy}

Fluorescence spectra were acquired using a home made setup based on IHR-320 spectrofluorometer (Horiba) coupled with a SR-830 lock-in amplifier (Standford Research System). The excitation of colloidal CdTe QDs was performed at 310 $\mathrm{nm}$ through a deep UV Mounted LED (Thorlabs) to avoid direct excitation of FA and make FRET detection easier. The fluorescence emission was collected in conventional right angle geometry. Squarred PMMA cuvettes of $1 \mathrm{~cm}$ optical path length were used.

\subsection{UV-visible spectroscopy}

UV-visible absorbance spectra of colloidal solutions were recorded using a Cary5000 spectrophotometer (Agilent) in transmission through the same PMMA cuvettes as those used for fluorescence spectroscopy. Each absorbance curve was obtained by differential measurements using Milli-Q water as a reference.

\subsection{Dynamic light scattering and zetametry}

DLS and zeta potential measurements were performed on a Malvern instrument ZetaSizer ZEN3600 equipped with a $633 \mathrm{~nm}$ laser. The temperature was set at $25^{\circ} \mathrm{C}$. For measuring the zeta potential of QDs dispersed in Milli-Q water (at $30 \mu \mathrm{M}$ ), we used folded capillary cells from ZetaSizer Nano Series (Malvern).

\subsection{Gel electrophoresis}

Electrophoresis was performed on the base of the so-called Native PAGE technique, with $0.75 \mathrm{~mm}$ thick non-denaturing polyacrylamide gels. The $6 \%$ staking gel was prepared with $1.2 \mathrm{~mL}$ of polyacrylamide $(30 \mathrm{~g}$ of acrylamide and $7.5 \mathrm{~g}$ of bis-acrylamide for $100 \mathrm{~mL}), 1.5 \mathrm{~mL}$ of Tris $\left(\mathrm{C}_{4} \mathrm{H}_{11} \mathrm{NO}_{3}, 0.5 \mathrm{M}, \mathrm{pH} 6.8\right), 3.3$ $\mathrm{mL}$ of Milli-Q water, $6 \mu \mathrm{L}$ of TEMED $\left(\mathrm{C}_{6} \mathrm{H}_{16} \mathrm{~N}_{2}\right)$, and $60 \mu \mathrm{L}$ of APS $(10 \mathrm{~g}$ of ammonium persulfate, $\left(\mathrm{NH}_{4}\right)_{2} \mathrm{~S}_{2} \mathrm{O}_{8}$, for $\left.100 \mathrm{~mL}\right)$. The $10 \%$ separation gel was prepared with $3.3 \mathrm{~mL}$ of polyacrylamide, $2.5 \mathrm{~mL}$ of Tris (1.5 M, pH 8.8), 4.1 $\mathrm{mL}$ of Milli-Q water, $10 \mu \mathrm{L}$ of TEMED and $100 \mu \mathrm{L}$ of APS.

The electrophoresis experiment was conducted under a voltage of $120 \mathrm{~V}$ (during 90 minutes) using Mini-PROTEAN 3 Cell (BioRad) with a TG-HCl migration buffer ( $\mathrm{pH} 8.3$ ) made of Tris (10g, PUFFERAN > 99.9\%, Carl Roth) and Glycin (57.6g, PUFFERAN > 99.9\%, Carl Roth) in 2L Milli-Q water. 
In each well, $20 \mu \mathrm{L}$ of the sample to analyse was added, mixed with $10 \mu \mathrm{L}$ of a solution made of $2.5 \mathrm{~mL}$ of Tris (0.5 M, pH 6.8), $4.0 \mathrm{~mL}$ of Glycerol (> 99\%), $1 \mathrm{~mL}$ of bromophenol $(0.15 \% \mathrm{w} / \mathrm{v})$ and $5 \mathrm{~mL}$ of Milli-Q water.

\subsection{Reproducibility and precision}

The precision on the wavelengths of the fluorescence band maxima is $\pm 1 \mathrm{~nm}$. The relative errors on the fluorescence signals are typically $2 \%$. The corresponding error bars are not drawn on the figures for clarity reasons.

\section{Acknowledgment}

J.H. and L.D. acknowledge the "Fonds de la Recherche Scientifique - FRS" for financial support. T.N. and C.H. acknowledge the funding from the International Scientific Cooperation Program of the CNRS (Centre National de la Recherche Scientifique) under Grant agreement number PICS07339. The authors acknowledge Dr. E. Brun (University of Paris-Sud, Université Paris-Saclay, Laboratoire de Chimie Physique, CNRS, 91405 Orsay, France) for her technical assistance on the DLS setup, and Dr. A. Brans (Laboratory of Enzymology and Protein Folding, Center for Protein Engineering (CIP), University of Liege, Quartier Agora, Allée du six Août 11, B6a, 4000 Liège, Belgium) for fruitful scientific discussions.

\section{Keywords}

Biochemical functionalization, biosensors, fluorescence spectroscopy, FRET, quantum dots.

\section{TOC}

Luminescent quantum dots prove to efficiently transfer their energy to fluorescent molecular probes when they are previously aggregated (see picture 8), which can lead to a new paradigm for the design of FRET-based biosensors.

\section{References}

[1] Xudong Fan, Ian M. White, Siyka I. Shopova, Hongying Zhu, Jonathan D. Suter, and Yuze Sun. Sensitive optical biosensors for unlabeled targets: A review. Anal. Chim. Acta, 620(1-2):8-26, 2008.

[2] Josiane P. Lafleur, Alexander Jönsson, Silja Senkbeil, and Jörg P. Kutter. Recent advances in lab-on-a-chip for biosensing applications. Biosens. Bioelectron., 76:213-233, 2016. 
[3] Aaron R. Clapp, Igor L. Medintz, J. Matthew Mauro, Brent R. Fisher, Moungi G. Bawendi, and Hedi Mattoussi. Fluorescence Resonance Energy Transfer between Quantum Dot Donors and Dye-Labeled Protein Acceptors. J. Am. Chem. Soc., 126(1):301-310, 2004.

[4] Jingyu Shi, Feng Tian, Jing Lyu, and Mo Yang. Nanoparticle based fluorescence resonance energy transfer (FRET) for biosensing applications. $J$. Mater. Chem. B, 3(35):6989-7005, 2015.

[5] Igor L. Medintz and Hedi Mattoussi. Quantum dot-based resonance energy transfer and its growing application in biology. Phys. Chem. Chem. Phys., 11(1):17-45, 2009.

[6] Aaydha C Vinayaka and Munna S Thakur. Facile synthesis and photophysical characterization of luminescent CdTe quantum dots for Forster resonance energy transfer based immunosensing of staphylococcal enterotoxin B. Luminescence, 28(6):827-835, 2013.

[7] Jagjit Nanda, Sergei A Ivanov, Marc Achermann, Ilya Bezel, Andrei Piryatinski, and Victor I Klimov. Light Amplification in the Single-Exciton Regime Using Exciton - Exciton Repulsion in Type-II Nanocrystal Quantum Dots. J. Phys. Chem. C, 111(42):15382-15390, 2007.

[8] Ryan R Cooney, Samuel L Sewall, D M Sagar, and Patanjali Kambhampati. Gain Control in Semiconductor Quantum Dots via State-Resolved Optical Pumping. Phys. Rev. Lett., 102(12):127404-127407, 2009.

[9] Kais Boujdaria and Omar Zitouni. Energy-band structure of CdTe and Si : a $\mathrm{sp}^{3}\left(\mathrm{~s}^{*}\right)^{2}$ k.p model. Solid State Commun., 129(3):205-210, 2004.

[10] Sameer Sapra, N Shanthi, and D D Sarma. Realistic tight-binding model for the electronic structure of II-VI semiconductors. Phys. Rev. B, 66(20):205202, 2002.

[11] Sameer Sapra and D D Sarma. Evolution of the electronic structure with size in II-VI semiconductor nanocrystals. Phys. Rev. B, 69(12):125304125310, 2004.

[12] Haiguang Zhao, Daniele Benetti, Lei Jin, Yufeng Zhou, Federico Rosei, and Alberto Vomiero. Absorption Enhancement in "Giant" Core/Alloyed-Shell Quantum Dots for Luminescent Solar Concentrator. Small, 12(38):5354$5365,2012$.

[13] Xiyan Li, Yong-Biao Zhao, Fengjia Fan, Larissa Levina, Min Liu, Rafael Quitero-Bermudez, Xiwen Gong, James Fan, Zhenyu Yang, Sjoerd Hoogland, Oleksandr Voznyy, Zheng-Hong Lu, and Edward H Sargent. Bright colloidal quantum dot light-emitting diodes enabled by efficient chlorination. Nat. Photonics, 12(3):159-164, 2018. 
[14] Thomas Noblet, Laurent Dreesen, S Boujday, C Méthivier, Bertrand Busson, A Tadjeddine, and Christophe Humbert. Semiconductor quantum dots reveal dipolar coupling from exciton to ligand vibration. Commun. Chem., 1(1):76, 2018.

[15] Shao-wen Cao, Yu-peng Yuan, Jun Fang, Mohammad Mehdi, Freddy Y C Boey, James Barber, Say Chye, Joachim Loo, and Can Xue. In-situ growth of CdS quantum dots on g-C $3 \mathrm{~N} 4$ nanosheets for highly efficient photocatalytic hydrogen generation under visible light irradiation. Int. J. Hydrogen Energy, 38(3):1258-1266, 2013.

[16] Esther Groeneveld, Christophe Delerue, Guy Allan, Yann-michel Niquet, and Celso De Mello Donega. Size Dependence of the Exciton Transitions in Colloidal CdTe Quantum Dots. J. Phys. Chem. C, 116(43):21160-23167, 2012.

[17] Aaron R. Clapp, Igor L. Medintz, and Hedi Mattoussi. Frster resonance energy transfer investigations using quantum-dot fluorophores. ChemPhysChem, 7:47-57, 2006.

[18] Marya Hardzei, Mikhail Artemyev, Michael Molinari, Michel Troyon, Alyona Sukhanova, and Igor Nabiev. Comparative efficiency of energy transfer from cdse?zns quantum dots or nanorods to organic dye molecules. ChemPhysChem, 13:330-335, 2012.

[19] I. V. Martynenko, A. P. Litvin, F. Purcell-Milton, A. V. Baranov, A. V. Fedorov, and Y. K. Gun'Ko. Application of semiconductor quantum dots in bioimaging and biosensing. J. Mater. Chem. B, 5(33):6701-6727, 2017.

[20] Christina Marie Tyrakowski and Preston Todd Snee. A primer on the synthesis, water-solubilization, and functionalization of quantum dots, their use as biological sensing agents, and present status. Phys. Chem. Chem. Phys., 16(3):837-855, 2014.

[21] Lifang Shi, Vania De Paoli, Nitsa Rosenzweig, and Zeev Rosenzweig. Synthesis and application of quantum dots FRET-based protease sensors. $J$. Am. Chem. Soc., 128(32):10378-10379, 2006.

[22] Gregory P. Mitchell, Chad A. Mirkin, and Robert L. Letsinger. Programmed assembly of DNA functionalized quantum dots. J. Am. Chem. Soc., 121(35):8122-8123, 1999.

[23] L. Dyadyusha, H. Yin, S. Jaiswal, T. Brown, J. J. Baumberg, F. P. Booy, and T. Melvin. Quenching of CdSe quantum dot emission, a new approach for biosensing. Chem. Commun., 0(25):3201-3203, 2005.

[24] Junghan Lee, Youngseon Choi, Junwon Kim, Eunjung Park, and Rita Song. Positively charged compact quantum dot?dna complexes for detection of nucleic acids. ChemPhysChem, 10:806-811, 2009. 
[25] A. C. Vinayaka and M. S. Thakur. Focus on quantum dots as potential fluorescent probes for monitoring food toxicants and foodborne pathogens. Anal. Bioanal. Chem., 397(4):1445-1455, 2010.

[26] Juan B. Blanco-Canosa, Miao Wu, Kimihiro Susumu, Eleonora Petryayeva, Travis L. Jennings, Philip E. Dawson, W. Russ Algar, and Igor L. Medintz. Recent progress in the bioconjugation of quantum dots. Coord. Chem. Rev., 263-264(1):101-137, 2014.

[27] Ute Resch-Genger, Markus Grabolle, Sara Cavaliere-Jaricot, Roland Nitschke, and Thomas Nann. Quantum dots versus organic dyes as fluorescent labels. Nat. Methods, 5(9):763-775, 2008.

[28] W. Russ Algar and Ulrich J. Krull. Luminescence and stability of aqueous thioalkyl acid capped $\mathrm{CdSe} / \mathrm{ZnS}$ quantum dots correlated to ligand ionization. ChemPhysChem, 8(4):561-568, 2007.

[29] H. Tetsuo Uyeda, Igor L. Medintz, Jyoti K. Jaiswal, Sanford M. Simon, and Hedi Mattoussi. Synthesis of compact multidentate ligands to prepare stable hydrophilic quantum dot fluorophores. J. Am. Chem. Soc., 127(11):3870-3878, 2005.

[30] Bing C. Mei, Kimihiro Susumu, Igor L. Medintz, James B. Delehanty, T. J. Mountziaris, and Hedi Mattoussi. Modular poly(ethylene glycol) ligands for biocompatible semiconductor and gold nanocrystals with extended $\mathrm{pH}$ and ionic stability. J. Mater. Chem., 18(41):4949, 2008.

[31] Kimihiro Susumu, Bing C. Mei, and Hedi Mattoussi. Multifunctional ligands based on dihydrolipoic acid and polyethylene glycol to promote biocompatibility of quantum dots. Nat. Protoc., 4(3):424-436, 2009.

[32] Andrew M. Smith, Hongwei Duan, Matthew N. Rhyner, Gang Ruan, and Shuming Nie. A systematic examination of surface coatings on the optical and chemical properties of semiconductor quantum dots. Phys. Chem. Chem. Phys., 8(33):3895-3903, 2006.

[33] Yue Zhang, Haiyan Zhang, Jeff Hollins, Michael E. Webb, and Dejian Zhou. Small-molecule ligands strongly affect the Förster resonance energy transfer between a quantum dot and a fluorescent protein. Phys. Chem. Chem. Phys., 13(43):19427-19436, 2011.

[34] Greg T. Hermanson. Bioconjugate Techniques. Academic Press, third edition, 2013.

[35] Dorota Bartczak and Antonios G. Kanaras. Preparation of peptidefunctionalized gold nanoparticles using one pot edc/sulfo-nhs coupling. Langmuir, 27:10119-10123, 2011.

[36] Zenon Grabarek and John Gergely. Zero-length crosslinking procedure with the use of active esters. Anal. Biochem., 185(1):131-135, feb 1990. 
[37] W. Russ Algar, Duane E. Prasuhn, Michael H. Stewart, Travis L. Jennings, Juan B. Blanco-Canosa, Philip E. Dawson, and Igor L. Medintz. The Controlled Display of Biomolecules on Nanoparticles: A Challenge Suited to Bioorthogonal Chemistry. Bioconjug. Chem., 22(5):825-858, 2011.

[38] Regina Bilan, Fabrice Fleury, Igor Nabiev, and Alyona Sukhanova. Quantum dot surface chemistry and functionalization for cell targeting and imaging. Bioconjug. Chem., 26(4):609-624, 2015.

[39] Jonathan Britton, Edith Antunes, and Tebello Nyokong. Fluorescence quenching and energy transfer in conjugates of quantum dots with zinc and indium tetraamino phthalocyanines. J. Photochem. Photobiol. A Chem., 210(1):1-7, 2010.

[40] Preston T. Snee. The Role of Colloidal Stability and Charge in Functionalization of Aqueous Quantum Dots. Acc. Chem. Res., 51(11):2949-2956, 2018 .

[41] W. Russ Algar, Anthony J. Tavares, and Ulrich J. Krull. Beyond labels: A review of the application of quantum dots as integrated components of assays, bioprobes, and biosensors utilizing optical transduction. Anal. Chim. Acta, 673(1):1-25, 2010.

[42] H. Mattoussi, J. Matthew Mauro, E. R. Goldman, G. P. Anderson, V. C. Sundar, F. V. Mikulec, and M. G. Bawendi. Self-assembly of CdSe-ZnS quantum dot bioconjugates using an engineered recombinant protein. $J$. Am. Chem. Soc., 122(49):12142-12150, 2000.

[43] Zhijun Ning, Matyas Molnár, Yun Chen, Peter Friberg, Liming Gan, Hans Ågren, and Ying Fu. Role of surface ligands in optical properties of colloidal CdSe/CdS quantum dots. Phys. Chem. Chem. Phys., 13(13):5848-5854, 2011.

[44] Aaydha C. Vinayaka and Munna S. Thakur. Photoabsorption and resonance energy transfer phenomenon in CdTe-protein bioconjugates: An insight into QD-biomolecular interactions. Bioconjug. Chem., 22(5):968-975, 2011.

[45] Hongyan Shen, Ali M. Jawaid, and Preston T. Snee. Poly(ethylene glycol) carbodiimide coupling reagents for the biological and chemical functionalization of water-soluble nanoparticles. ACS Nano, 3:915-923, 2009.

[46] Dale M. Willard, Lori L. Carillo, Jaemyeong Jung, and Alan Van Orden. CdSe-ZnS Quantum Dots as Resonance Energy Transfer Donors in a Model Protein-Protein Binding Assay. Nano Lett., 1(9):469-474, 2001.

[47] Andrea Schroedter, Horst Weller, Ramon Eritja, William E. Ford, and Jurina M. Wessels. Biofunctionalization of Silica-Coated CdTe and Gold Nanocrystals. Nano Lett., 2(12):1363-1367, 2002. 
[48] Fadi Aldeek, Lavinia Balan, Jacques Lambert, and Raphaël Schneider. The influence of capping thioalkyl acid on the growth and photoluminescence efficiency of CdTe and CdSe quantum dots. Nanotechnology, 19:475401, 2008.

[49] Samuel J Clarke, C A Hollmann, Faisal A Aldaye, and Jay L Nadeau. Effect of Ligand Density on the Spectral, Physical, and Biological Characteristics of CdSe / ZnS Quantum Dots. Bioconjug. Chem., 19(2):562-568, 2008.

[50] Craig F. Bohren and Donald R. Huffman. Absorption and Scattering of Light by Small Particles. Wiley, 1983.

[51] Thomas Noblet, Laurent Dreesen, Julie Hottechamps, and Christophe Humbert. A global method for handling fluorescence spectra at high concentration derived from the competition between emission and absorption of colloidal CdTe quantum dots. Phys. Chem. Chem. Phys., 19(39):2655926565, 2017.

[52] C R Kagan, C B Murray, and M G Bawendi. Long-range resonance transfer of electronic excitations in close-packed CdSe quantum-dot solids. Phys. Rev. B, 54(12):8633-8643, 1996.

[53] Sander F. Wuister, Rolf Koole, Celso Mello De Donegá, and Andries Meijerink. Temperature-dependent energy transfer in cadmium telluride quantum dot. J. Phys. Chem. B, 109(12):5504-5508, 2005.

[54] T. Franzl, D. S. Koktysh, T. A. Klar, A. L. Rogach, J. Feldmann, and N. Gaponik. Fast energy transfer in layer-by-layer assembled CdTe nanocrystal bilayers. Appl. Phys. Lett., 84(15):2904-2906, 2004.

[55] S. A. Crooker, J. A. Hollingsworth, S. Tretiak, and V. I. Klimov. Spectrally Resolved Dynamics of Energy Transfer in Quantum-Dot Assemblies: Towards Engineered Energy Flows in Artificial Materials. Phys. Rev. Lett., 89(18):18-21, 2002.

[56] C R Kagan, C B Murray, M Nirmal, and M G Bawendi. Electronic energy transfer in CdSe quantum dot solids. Phys. Rev. Lett., 76(9):1517-1520, 1996.

[57] Sergiy Mayilo, Jan Hilhorst, Andrei S. Susha, Cornelia Höhl, Thomas Franzi, Thomas A. Klar, Andrey L. Rogach, and Jochen Feldmann. Energy transfer in solution-based clusters of CdTe nanocrystals electrostatically bound by calcium ions. J. Phys. Chem. C, 112(37):14589-14594, 2008.

[58] Kenny F. Chou and Allison M. Dennis. Förster resonance energy transfer between quantum dot donors and quantum dot acceptors. Sensors, 15(6):13288-13325, 2015. 
[59] Jinjie Wang, Xiangyi Huang, Lingao Ruan, Tao Lan, and Jicun Ren. Size exclusion chromatography as a universal method for the purification of quantum dots bioconjugates. Electrophoresis, 34(12):1764-1771, 2013.

[60] Rudolf Gesztelyi, Judit Zsuga, Adam Kemeny-Beke, Balazs Varga, Bela Juhasz, and Arpad Tosaki. The Hill equation and the origin of quantitative pharmacology. Arch. Hist. Exact Sci., 66:427-438, 2012.

[61] Jody L Swift and David T Cramb. Nanoparticles as Fluorescence Labels : Is Size All that Matters? Biophys. J., 95(2):865-876, 2008.

[62] Helmut Hinterwirth, Stefanie Kappel, Thomas Waitz, Thomas Prohaska, Wolfgang Lindner, and Michael Lämmerhofer. Terms of Use Quantifying Thiol Ligand Density of Self-Assembled Monolayers on Gold Nanoparticles by Inductively Coupled Plasma À Mass Spectrometry. ACS Nano, 7(2):1129-1136, 2013. 


\begin{tabular}{cc}
\hline Label & Biotin/QD ratio \\
\hline$B_{0}$ & $2.10^{0}$ \\
$B_{1}$ & $2.10^{1}$ \\
$B_{2}$ & $2.10^{2}$ \\
$B_{3}$ & $2.10^{3}$ \\
$B_{4}$ & $2.10^{4}$ \\
\hline
\end{tabular}

Table 1: Labels assigned to the different ratios of number of biotin molecules for 1 CdTe QD used in this article.

\begin{tabular}{lccc}
\hline System & $N$ & $n$ & $k_{d}(\mu \mathrm{M})$ \\
\hline Aggregated-QD-based & 2.64 & 1.43 & 0.071 \\
Free-QD-based & 3.12 & 2.02 & 0.616 \\
\hline
\end{tabular}

Table 2: Fitting parameters extracted from Fig. 7 and Eq. (7).

(a)

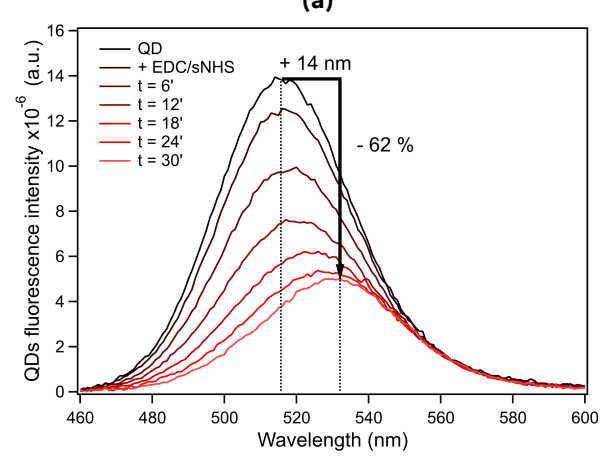

(b)

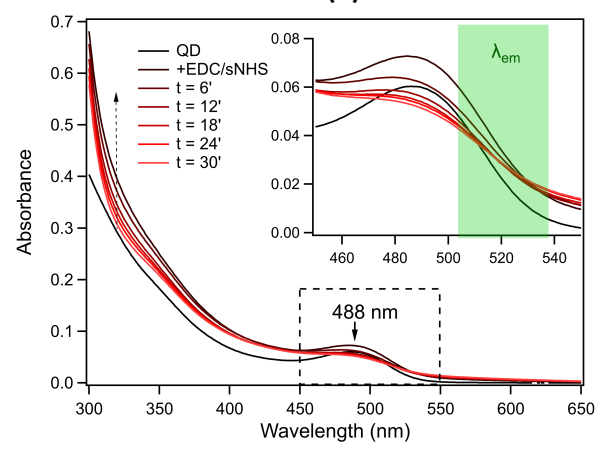

(c)

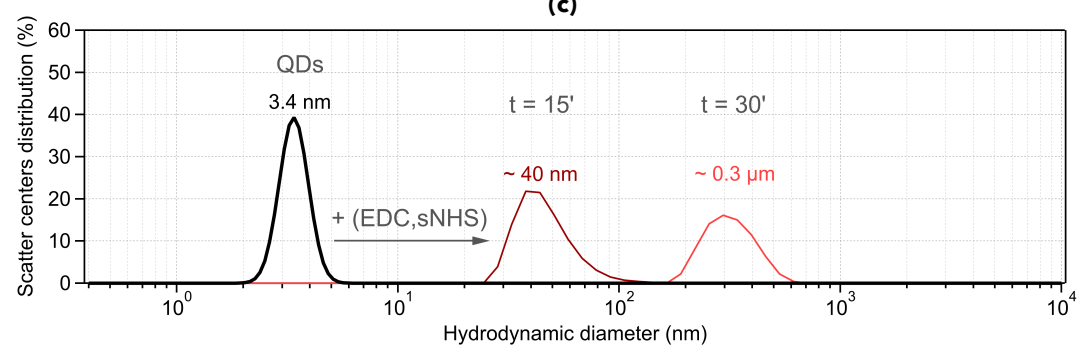

Figure 1: (a) Fluorescence spectra of colloidal CdTe QDs during the activation. The activation starts at the addition of EDC and sulfoNHS to QDs solution and lasts $30 \mathrm{~min}$ with a measurement every 6 minutes. (b) UV-visible absorption spectra of QDs during the activation step which starts at the addition of EDC and sulfoNHS to QDs and lasts 30 min with a measure every 6 minutes. (c) Results of DLS measurements performed on QDs (black) and activated QDs after 15 minutes (middle red) and 30 minutes (red). The sulfoNHS-to-QD ratio was set to $400: 1$ for all measurements. 


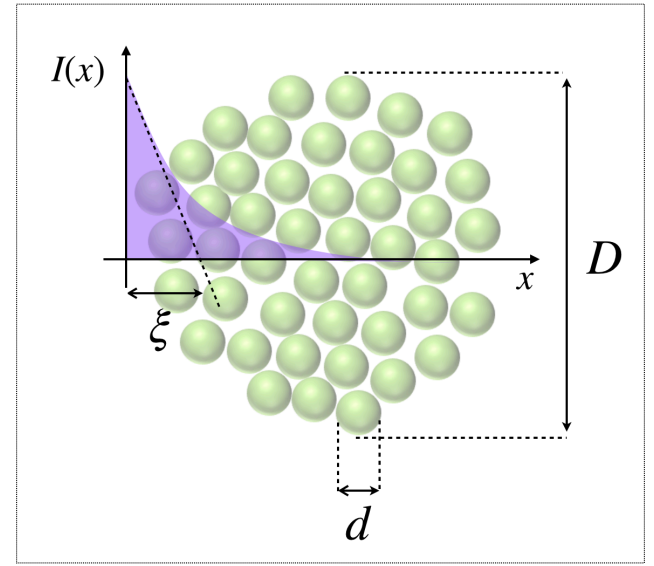

(a)

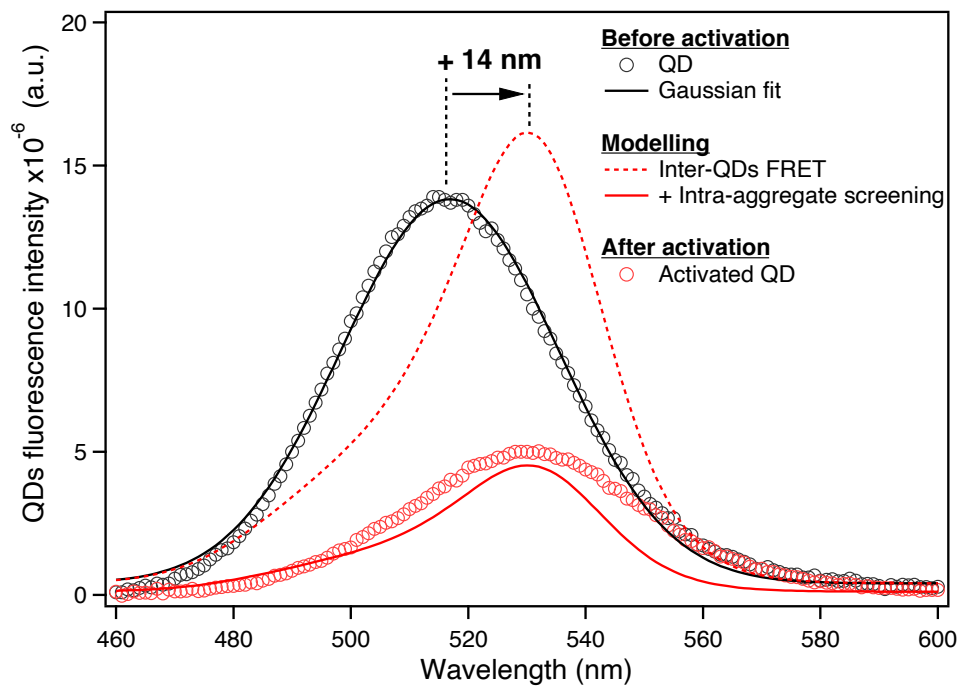

(b)

Figure 2: (a) Sketch of a QD aggregate and their characteristic lengths. (b) Fluorescence spectra of QDs before activation (black circles) and after (red circles) with a $400: 1$ sulfoNHS/QD ratio. The red lines correspond to the model we apply to account for inter-QDs FRET (dashed line) and intra-agglomerate screening (solid line). 

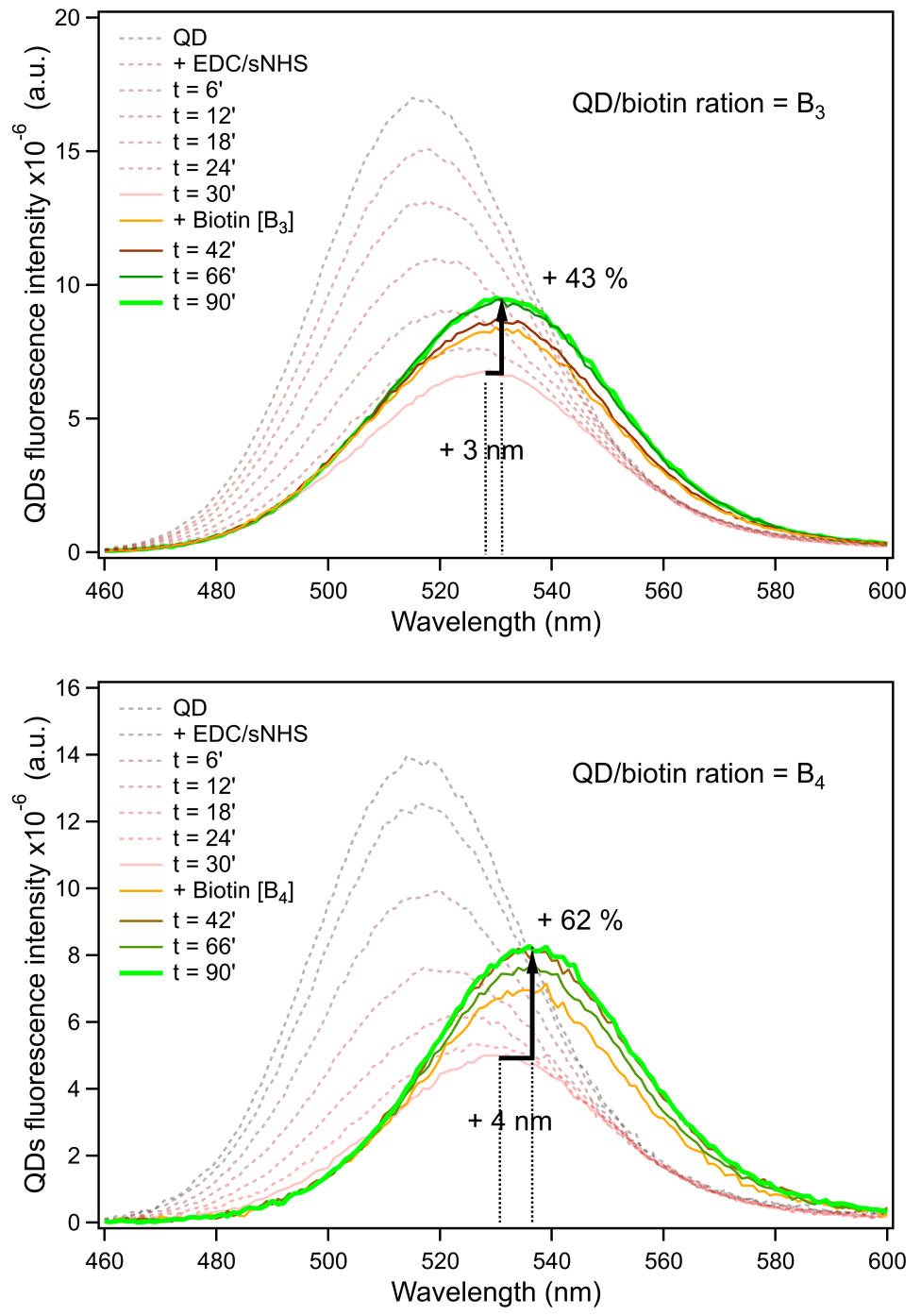

Figure 3: Fluorescence spectra of colloidal CdTe QDs during the biotinylation step (green tones). The biotinylation starts at the addition of biotin to activated QDs and lasts 60 min with a measurement every 12 minutes. The two biotin/QD ratios presented here are $B_{3}$ (up) and $B_{4}$ (down). 

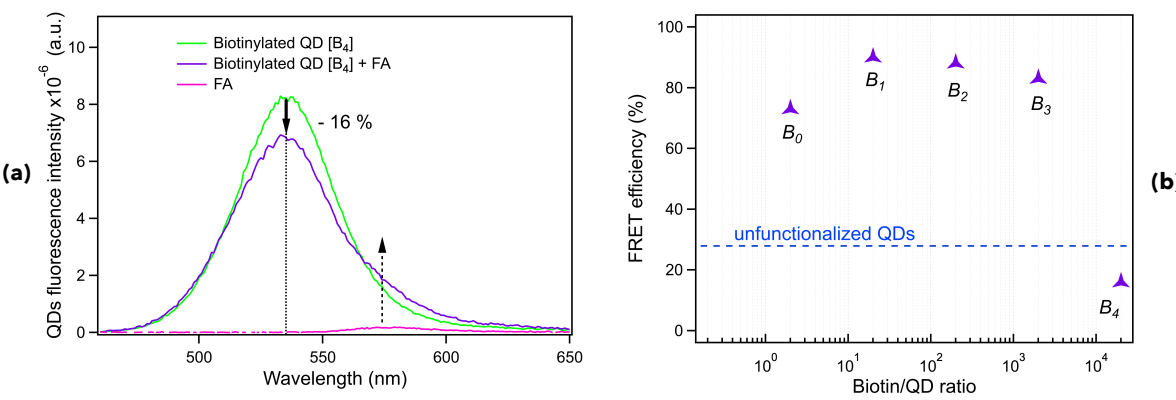

(b)
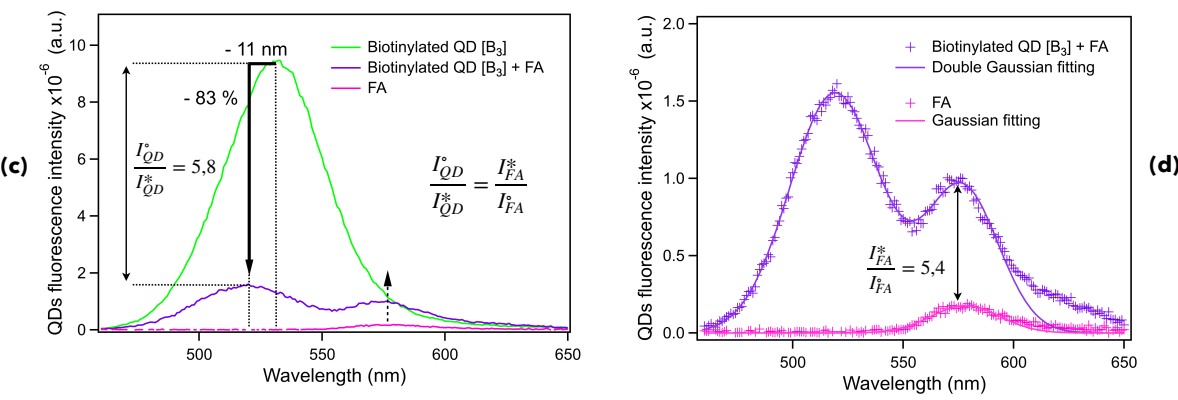

Figure 4: (a) Fluorescence spectra of FA (fuchsia), and of biotinylated QDs with FA (purple) or without FA (green). The sulfoNHS-to-QD ratio was set at $400: 1$ whereas the biotin-to-QD ratio was $B_{4}$. (b) FRET efficiency, computed from the loss of functionalized QDs fluorescence intensity, in the presence of a $1: 1 \mathrm{FA} / \mathrm{QD}$ ratio with respect to increasing biotin/QD ratio in the samples. The QDs were activated with a $400: 1$ sulfoNHS/QD ratio beforehand. (c) Fluorescence spectra of FA (fuchsia), and of biotinylated QDs with FA (purple) or without FA (green). The sulfoNHS-to-QD ratio was set at 400:1 whereas the biotin-to-QD ratio was $B_{3}$. (d) Zoom on the fluorescence spectra of FA (fuchsia) and biotinylated QDs with FA (purple) given in Fig. 4c. Here, the continuous lines correspond to Gaussian fits which enable to extract the ratio between the fluorescence intensities of FA, $I_{F A}^{\circ}$, without and, $I_{F A}^{*}$, with QD-based FRET. 


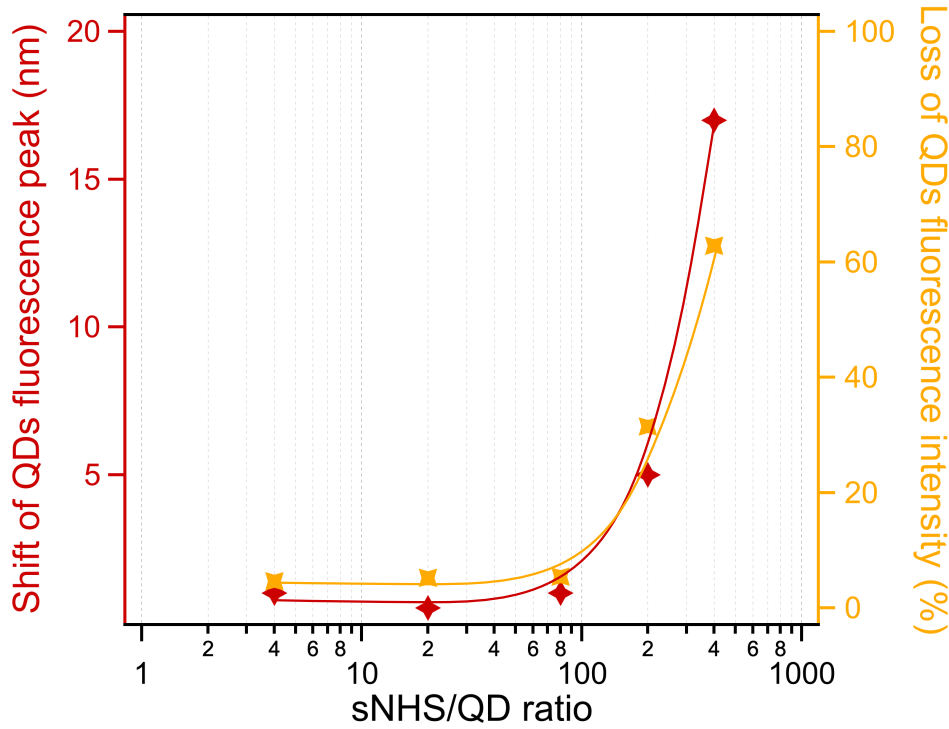

(a)

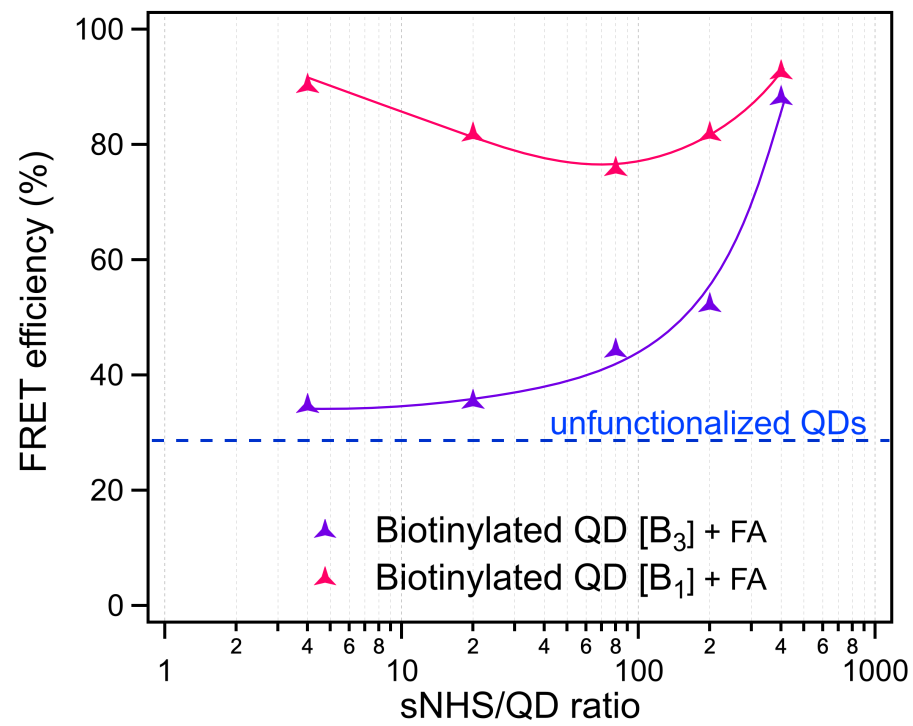

(b)

Figure 5: (a) Bathochromic shift (red) and intensity loss (yellow) of the fluorescence band of activated QDs with respect to sNHS/QD ratio. (b) FRET efficiency of functionalized QDs in the presence of a $1: 1 \mathrm{FA} / \mathrm{QD}$ ratio with respect to sNHS/QD ratio for QDs biotinylated with, first, a biotin/QD ratio $B_{3}$ (purple dots) and, second, a biotin/QD ratio $B_{1}$ (fuchsia dots). In both graphs, the lines correspond to trend curves. 


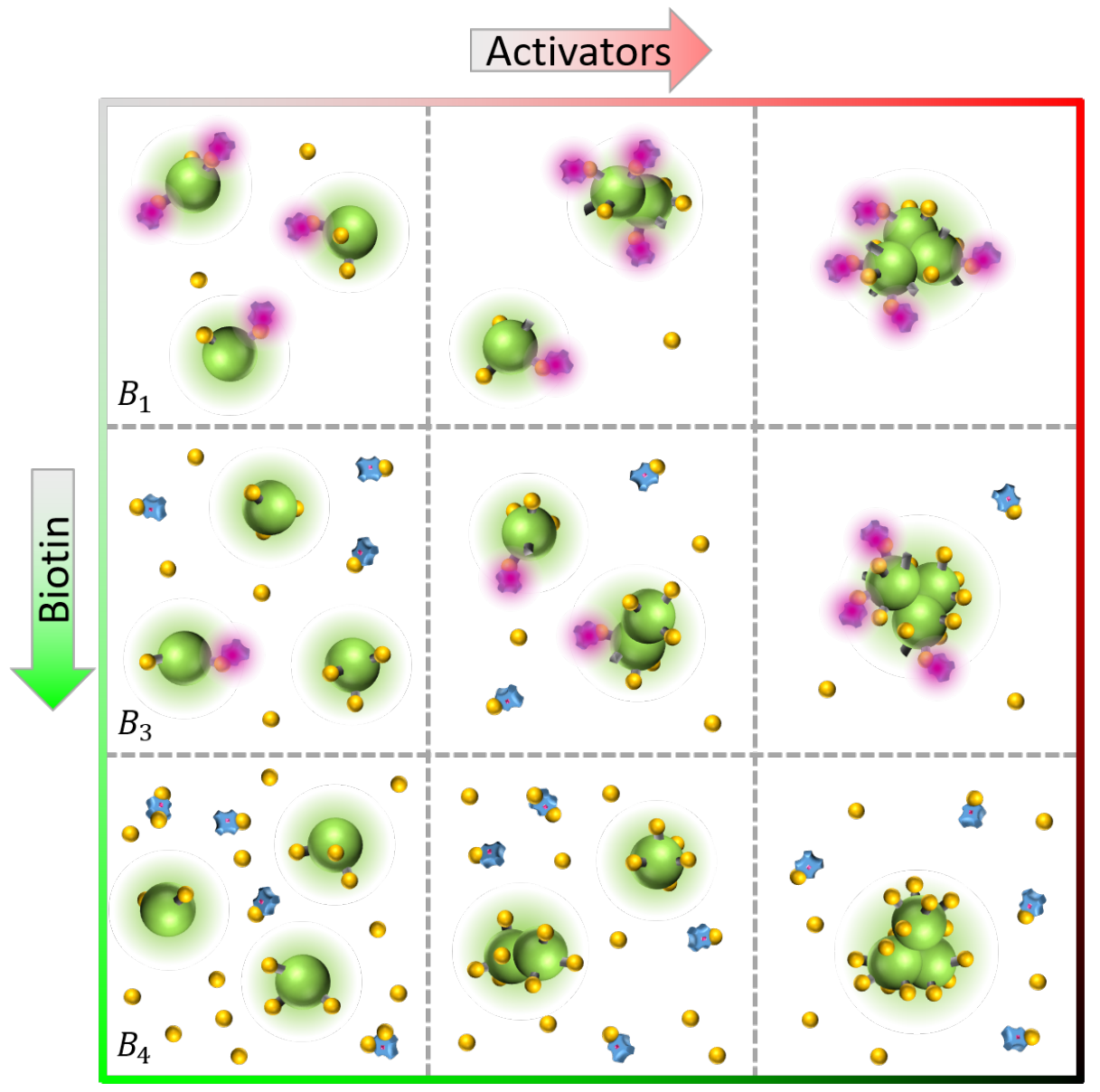

Figure 6: Sketch of the interactions between QDs and FA in solution according to the functionalization protocol. The green spheres represent the QDs whereas the yellow ones symbolize the biotin molecules. The blue crosses embody the streptavidin with a fluorophore represented by the fuchsia square on its top, forming together the FA. The QDs fluorescence appears as a green halo whereas the FA fluorescence is represented with a fuchsia halo. The latter occurs only if FA is closed enough from a QD due to FRET. The increase of activators and biotin concentrations are symbolized with red and green arrows, respectively. 


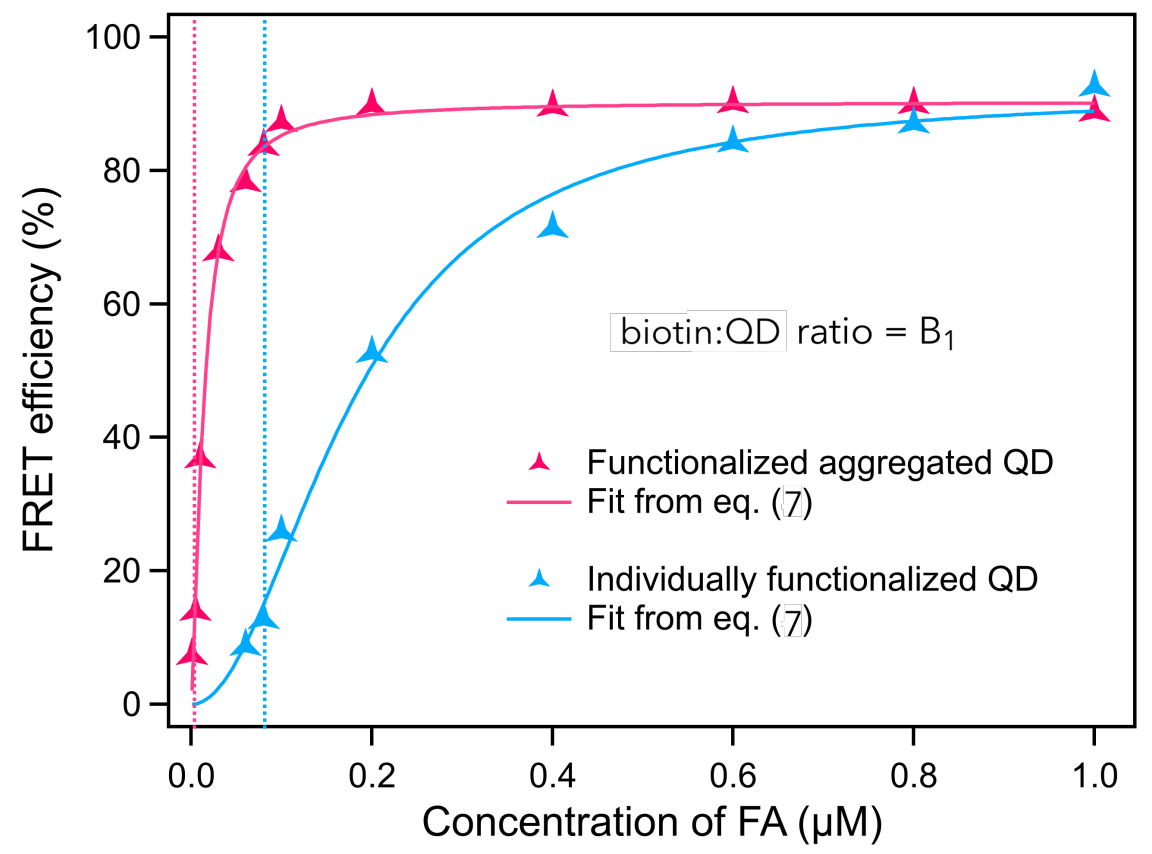

Figure 7: FRET efficiency with respect to FA concentration for aggregatedQD-based (fuchsia) and free-QD-based systems (blue). The detection limit associated to each system is drawn as a vertical dashed line in their respective colors.

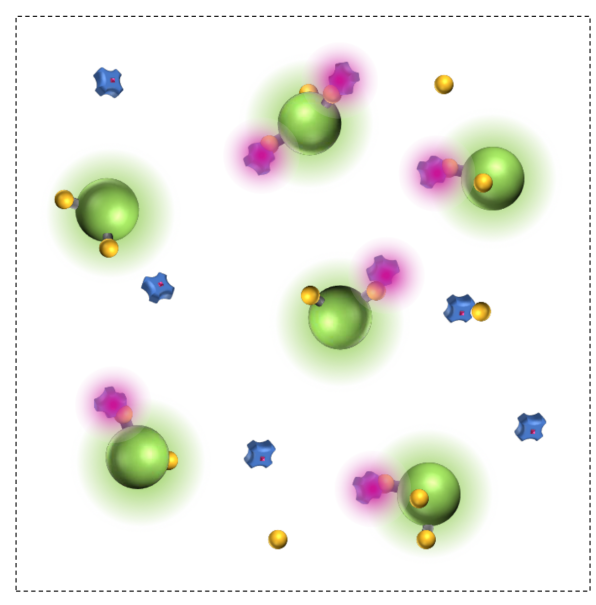

Free-QD-based system

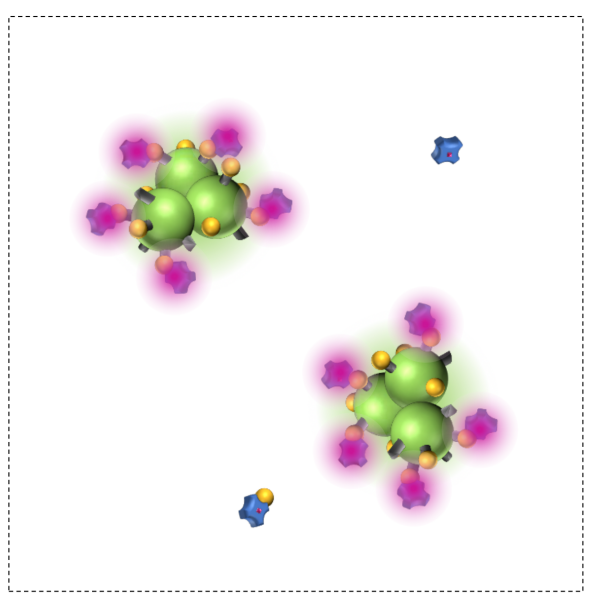

Aggregated-QD-based system

Figure 8: TOC figure. 